\title{
Tubular micromotors: from microjets to spermbots
}

\author{
Veronika Magdanz ${ }^{1 *}$, Maria Guix ${ }^{1 *}$ and Oliver G Schmidt ${ }^{1,2,3^{*}}$
}

\begin{abstract}
In the last decade, it was demonstrated that tubular micromotors are of potential interest for environmental, sensing, and medical applications. Even though catalytic micromotors rely on toxic fuel for their propulsion, many proof-of-concept applications were shown. Currently, the research community of micromotors is searching for biocompatible fuel solutions in order to extend the field of applications to tasks in physiological conditions. This short review gives an overview over the advances in the field of tubular micromotors explaining the different fabrication methods, fuels, and applications. The article points out the utilization of catalytic microjets in sensing, medical, and environmental applications, as well as the journey towards biocompatible tubular motors driven by motile sperm cells.
\end{abstract}

Keywords: Microjets; Rolled-up; Microtubes; Micromotors; Spermbot; Nanotechnology; Microfabrication

\section{Review}

The development of tiny machines that are able to function on the microscale to perform various tasks are of special interest nowadays. This review focuses on the state of the art in the field of tubular micromotors spanning from catalytically driven microjets to hybrid micromotors propelled by motile sperm cells. The article stresses especially the fabrication methods and describes control mechanisms, fuels, applications, and further progress towards biocompatible alternatives that have ensued in the recent years. This article is only reviewing tubular micromotors and does not include micromotors of other shapes, such as spheres, rods, helices, or other designs, being out of scope of this short review.

There are two distinct ways to fabricate tubular micromotors: roll-up of nanomembranes and electrodeposition into the pores of membranes, being used as template. Although the fabrication procedures strongly differ, both methods offer a reliable mass production of catalytic microjets. The following paragraphs describe rolled-up nanotech and template-based deposition of microjets in detail and also present a comparative table

\footnotetext{
* Correspondence: v.magdanz@ifw-dresden.de; m.guix.noguera@ifw-dresden.de; o.schmidt@ifw-dresden.de

${ }^{1}$ Institute for Integrative Nanosciences, Leibniz Institute for Solid State and Materials Research Dresden, 01069 Dresden, Germany

${ }^{2}$ Material Systems for Nanoelectronics, Chemnitz University of Technology, 09126 Chemnitz, Germany

Full list of author information is available at the end of the article
}

(Table 1). In addition, the proof-of-concept applications that were demonstrated in the last decade for rolled-up and template-based microjets will be discussed.

\section{Rolled-up nanotech: 'a tool for many trades'}

Rolled-up nanotech is a method of microfabrication that has opened a wide field of applications of microtubes $[1,2]$. Recent publications demonstrate the utility of rolledup microtubes, e.g., as highly sensitive sensors [3,4], for microbatteries [5], for transistors [6], and as a tool to study cells in confinement $[7,8]$. Rolled-up nanotech was also utilized to fabricate the first tubular micromotors $[2,9]$ with an inner platinum surface for bubble propulsion by catalytic decomposition of hydrogen peroxide.

\section{Inorganic microjets from rolled-up nanomembranes}

Rolled-up nanotech offers a versatile design of tubular micromotors. Geometrical parameters such as the radius, length, and shape of the micromotors can be varied, on the one hand by changing the pattern dimensions that are used for the deposition of nanomembranes on the substrate and on the other hand by the deposition angle, rate, and thickness of the material. This method can be used to fabricate conical or rather cylindrical microtubes of lengths ranging from the nanometer [10] to millimeter scale [11]. Almost any material can be integrated into rolled-up microtubes, and therefore, the technology can

\section{穴}


Table 1 Comparative table of template-based electrodeposition and rolled-up nanotech as fabrication methods for tubular micromotors

\begin{tabular}{lll}
\hline & Template-based electrodeposition & Rolled-up nanotech \\
\hline Required & - Porous membranes as templates & - Photolithography \\
Suitable materials & - Electrodeposition setup & - Thin film deposition setup \\
Advantages & - Easily accessible method & - Materials compatible with vacuum deposition \\
& $\cdot$ Compact fabrication setup & - Wide range of materials \\
& & - Fabrication of tubular structures with wide range of length and diameter \\
& & possible \\
& & - Fine control of layer thickness down to nanometer precision \\
Limitations & - Tube shape preset by template pores & - Often requires vacuum deposition machine \\
& - Requires conductive outer layer & \\
& Release of tubes after dissolution of & \\
& membrane & \\
\hline
\end{tabular}

This table compares the two distinct fabrication methods for tubular micromotors according to the current state of the art. However, this might change in the future as the processes are optimized and developed further.

implement a variety of new functionalities into the tubular micromotors.

Rolled-up nanotech is based on the strain engineering of inorganic nanomembranes that roll into microtubes when they are released from a sacrificial layer by etching with organic solvents [2]. First of all, a substrate, most commonly a glass slide or silicon wafer, is spin-coated with a photoresist. After exposure to ultraviolet light through a photomask, the photoresist is developed with an etching agent. Left on the substrate are certain photoresist patterns, e.g., squares or circles, which are the base for depositing nanomembranes of certain sizes and shapes via electron beam evaporation. The electron beam releases atoms from the target material under high-vacuum conditions. These atoms solidify on the surface of the substrate and form a thin film. During glancing angle deposition, a narrow gap remains open at the far end of the patterned photoresist where no material is deposited. This narrow gap, also called 'window', is a point of attack for the etching solution that removes the photoresist. When the photoresist starts dissolving, nanomembranes roll up into tubes due to the internal strain as they release from the substrate (Figure 1). A certain thickness of each material, as well as a defined tube radius, can be achieved depending on the rate and time of deposition of each layer. An alternative to the etching of the sacrificial layer for achieving roll-up is the dry-releasing approach by thermal annealing using self-assembled metal nanodroplets [12].

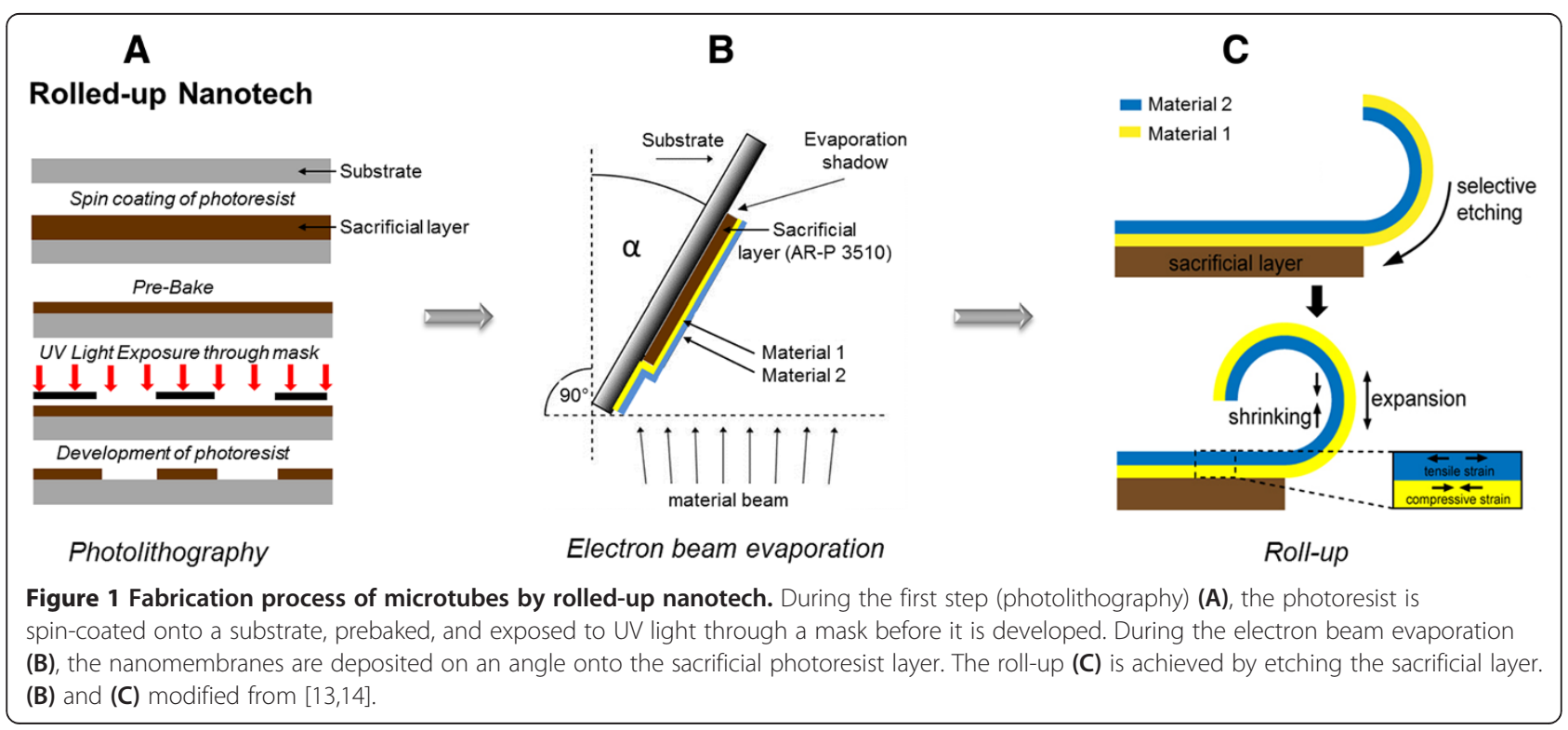


For the creation of tubular bubble-propelled microjets, an inner platinum surface is essential; therefore, a deposition of nanomembranes such as titanium (adhesion layer), chromium (induces additional strain), iron (for magnetic remote control), and platinum (catalytic surface) is chosen. An inner or outer gold nanomembrane can be integrated in the microjet's design by means of sputtering or evaporation techniques for its further functionalization. Immobilizations of molecules on gold surfaces are accomplished by surface chemistry methods such as self-assembling monolayers (SAMs) of thiols [15] and/or other chemical linkers that selectively attach to the active groups of the target molecule. This method provides the ability to pick up and transport particles or biological entities, leading to their isolation in complex samples (e.g. serum, urine, saliva). The first example of pick-up and delivery of single cells by tubular micromotors was demonstrated by Sanchez et al. [16]. The Pt microjets with an outer gold layer can also serve as sensors: some clear examples are the functionalization of such SAMs with single-strand DNA probe [17], anticarcinoembryonic antigen monoclonal antibody [18], or aptamers [19], permitting the selective capture of nucleic acids, cancer cells, or target proteins, respectively.

The incorporation of a magnetic nanomembrane provides the remote control via external magnetic fields [9]. When the tubular micromotors are magnetized by exposure to a permanent magnet, they act as compasses and sense the direction of the external magnetic field (Figure 2BIII) [20].
The magnetic layer also allows the utilization of the microtubes as micro-/nanodrillers [10,21]. In addition, the shape of the tubular micromotors can be altered to be slightly conical with a sharp tip when thin films are deposited on trapezoidal photoresist structures. This leads to the ability of the drillers to make incisions in cells and tissue [21]. It was demonstrated that microjets are powerful enough to swim in a controlled manner against flow in microfluidic channels [22,23]. Two- and three-dimensional (3D) closedloop control $[24,25]$ was achieved using a setup of electromagnetic coils that direct the magnetic field lines towards reference positions (Figure 2AII). This technique offers an accurate remote positioning and motion control of the microjets in 3D space. Light and temperature also serve as control parameters to change the speed of microjets during their operation [26]. Light diminishes locally the concentration of hydrogen peroxide, reducing the motility of the microjets in this area (Figure 2BI). This leads to a stopand-go motion of the microjets when light is applied sequentially. An increase in temperature up to $37^{\circ} \mathrm{C}$ also improves the catalytic decomposition of peroxide to oxygen and water and leads to an acceleration of the microjets (Figure 2BIIa) [27,28].

Furthermore, rolled-up micromotors can be fabricated from 20- to 200-nm-thick iron nanomembranes, which actively decompose organic pollutants in an acidic environment [29]. It was also shown that micromotors can be trapped in microchips containing chevron and heart-shaped structures without any external

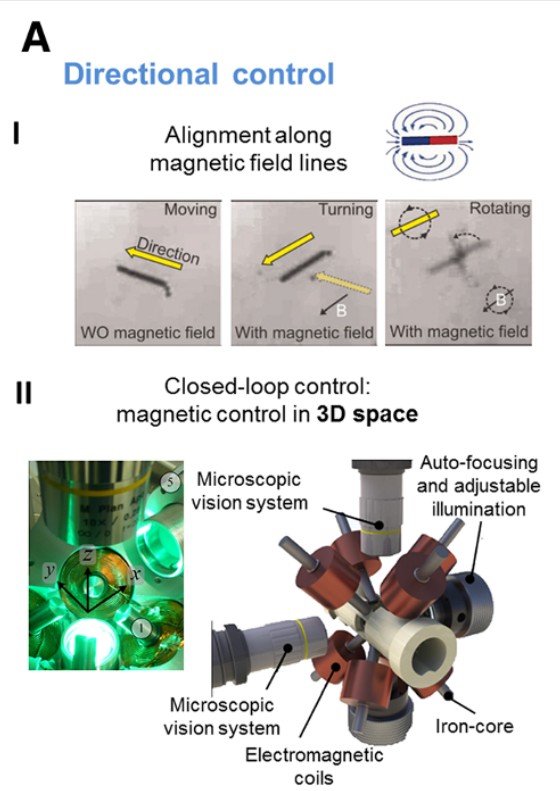

B Speed control
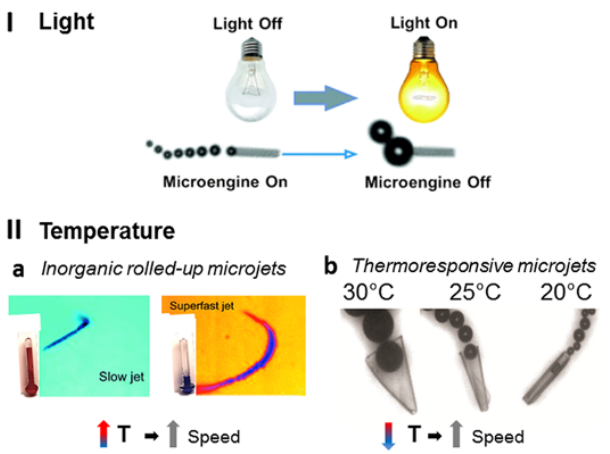

III Magnetic field

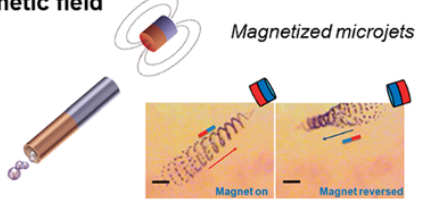

Figure 2 Control mechanisms for direction and speed of motion of microjets. (A) Directional control through alignment of microjets with external magnetic fields [9] (I) and closed-loop control of microjets in 3D by setup of eight electromagnetic coils [25] (II). (B) Speed control of microjets by changing light intensity [26] (I) or temperature [27,34] (II). A magnetic field can be used to attract and accelerate or repel and reverse the motion of magnetized microjets (III) [20]. 
mechanism due to their self-propulsion and can be used as an alternative system to concentrate components in the case of working with functionalized micromotors $[30,31]$. A particular treat of strain-engineered rolled-up micromotors is the integration of electronic functionality as envisioned in Mei et al. [32], rendering these promising engines for future fully autonomous and multi-functional microsystems.

\section{Stimuli-responsive rolled-up microjets based on thermoresponsive polymers}

Polymers are attractive materials because of their flexibility, transparency, and sensitivity to external stimuli. Stimuliresponsive polymer tubes were shown to open and close when a small temperature change is applied [33]. Polymeric bilayers were utilized to fabricate thermoresponsive microjets that fold and unfold upon temperature change [34]. Polycaprolactone as outer passive layer, poly(N-isopropylacrylamide) (PNIPAM) as middle thermo-active layer, and platinum as inner catalytic layer form the triple film of the microjet. PNIPAM swells below $28^{\circ} \mathrm{C}$ and shrinks above this temperature, so that a slight temperature difference leads to a change in the radius of the multilayer jet (Figure 2BIIb). By doing so, the microjet can be reversibly accelerated and stalled on demand, because the radius determines the bubble propulsion and, in turn, the speed of the microjet. Polymers are also, as mentioned above, easily functionalized with bioactive molecules. In addition, such flexible microjets have potential for studies of the mechanism of bubble generation inside tubular microjets and for biodegradable micromotors.

\section{Quick and easy: template-based microjets by electrodeposition}

Although the very first tubular micromotors fabricated by using electrochemical techniques were obtained by depositing platinum and gold onto a silver wire which was then subjected to further etching [35], breakthrough to much more attractive implementation came with the template-based tubular micromotors electrochemically deposited into porous membranes. The fabrication of template-based tubular microjets is based on sequentially depositing the materials of interest into a template (generally a tubularly or conically shaped porous membrane), followed by its dissolution and the release of the tubular micromotors. Such procedure is carried out by applying an electrical current that reduces cations from a desired material of the solution, leading to the deposition of such material onto the walls of the porous membrane.

The setup to perform such electrochemical depositions is composed by a counter electrode (i.e., platinum wire), a reference electrode (i.e., $\mathrm{Ag} / \mathrm{AgCl}$ electrode), and a working electrode, which is based on a conductive layer that is in contact with one face of the porous membrane (see Figure 3A). Both aluminum oxide membranes (AAO membranes) [36] and polycarbonate membranes [37] are used for such purposes so as to obtain tubular or conical microtubes, respectively. In both cases, it is
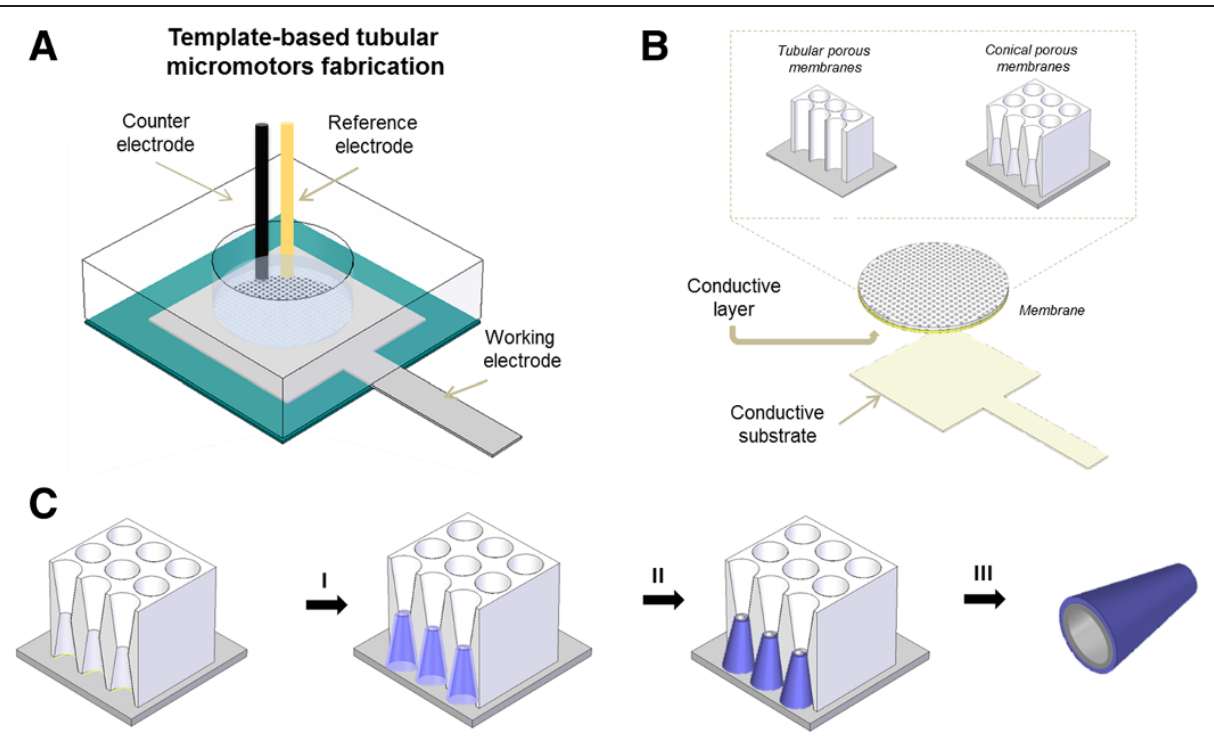

Figure 3 General schematic of an electrochemical plating cell and main steps to fabricate conical template-based tubular micromotors. (A) General schematic of an electrochemical plating cell and (B) zoom-in of the working electrode system, based on a conductive substrate in contact with the conductive face of the tubular or conical porous membrane. (C) Main steps followed to fabricate conical template-based tubular micromotors are deposition of the outer polymeric conductive layer (I), followed by the sequential deposition of the inner catalytic surface (II), and the final dissolution of the membrane (III) to release the micromotors. 
especially important to sputter one side of the membrane with a conductive metal layer so as to obtain the proper contact between the conductive substrate and the template (Figure 3B).

The fastest and most efficient mass production procedure to obtain conical micromotors of about $8 \mu \mathrm{m}$ in length and $1 \mu \mathrm{m}$ in diameter is based on the previous deposition of a polyaniline (PANI) conductive polymeric layer onto polycarbonate membranes with conically shaped pores (Figure 2AI), which will be further used as the conductive layer template for the next sequential electrodeposition steps. Therefore, the sequential electrochemical deposition of solid metals (i.e., platinum) will be performed on the previous conductive layer, leading to the fabrication of perfectly conical microjets embedded into the polycarbonate membrane. After removing the previously sputtered conductive layer by hand polishing with alumina slurry, the polycarbonate membrane is dissolved and the micromotors will be released (Figure 3C) [38].

Among the different possibilities to deposit the first conductive template, some alternatives to PANI had been reported. Polypyrrole (PPy) or poly(3,4-ethylenedioxythiophene) (PEDOT) suits the requirements to obtain the outer layer (Figure 4AI) [37]. Zhao et al. had also reported a similar procedure, where the first deposited layer on the walls of the template (conductive layer template) is based on a copper layer (Figure 4AII) [39]. It is of special interest that the outer layer of the micromotors can be directly functionalized with certain functional groups inherently present in the polymer, as the carboxylic PEDOT used for on-chip immunoassays [40] or poly(3-aminophenylboronic acid) (PAPBA), which permits the recognition of yeast cells [41].

Two key parameters to properly manipulate the movement and the successful development of template-based microjets in environmental and sensing applications are their magnetic guidance and the functionalization of their outer surface. Such features are generally achieved by the integration of a magnetic layer (e.g. nickel (Ni) or iron (Fe) layer) and an external gold layer, which is obtained by sputtering a gold layer once the microjets are released from the template (covering only one side of the micromotor). In the case of polymeric outer layers, the Au layer is not required since they can be functionalized directly [40] or do not require any functionalization $[41,46]$.

Additionally, the use of ultrasound field has recently been introduced as fast speed control method. Ultrasound induces the disruption of normal bubble evolution and ejection and thereby changes the movement of PEDOT/Ni/Pt micromotors in a sharp and reproducible manner [47].

One of the intriguing features of template-based micromotors is their size, which is in the same order of certain analytes of interest, such as bacteria or cancer cells, allowing a clear visualization of the pick-up and transport events by using optical microscopy. By convenient functionalization of the outer surface of the template-based tubular micromotors, the selective capture of E. coli bacteria [48] and a complete on-chip immunoassay were achieved [40]. However, some reported configurations required no functionalization to perform
A Hydrogen peroxide

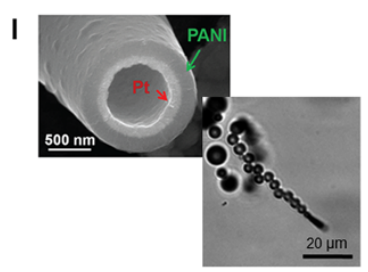

III

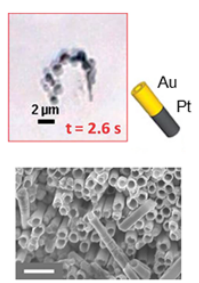

II

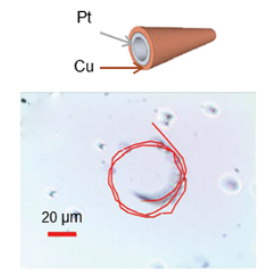

IV

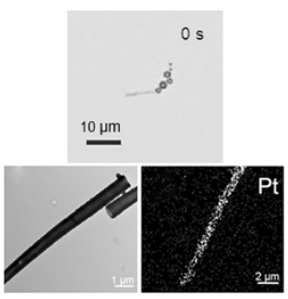

B Acidic conditions

I

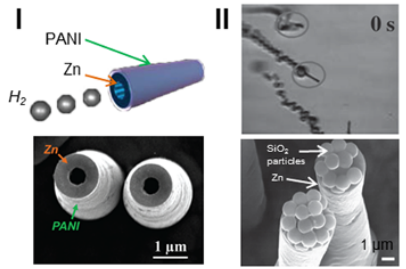

C Water diffusion
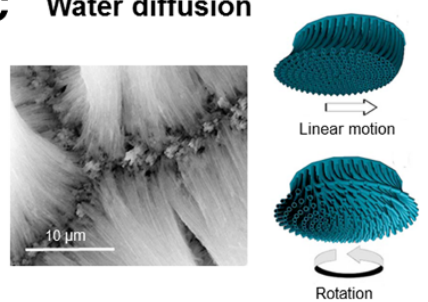

Figure 4 Template-based micromotors driven by chemical fuels. (A) Micromotors moving due to the decomposition of $\mathrm{H}_{2} \mathrm{O}_{2}$ at the platinum surface: PANI/Pt conical micromotors [38] (I), Cu/Pt conical micromotors [39] (II), tubular nanometric Au-Pt nanomotor [36] (III), and multilayer tubular nanomotors with a Pt inner surface [42] (IV). (B) Micromotors moving in the presence of acidic environment: PANI/Zn [43] (I) and fully loaded zinc micromotors [44] (II). (C) Linear and rotational movement of $\left(\mathrm{Pb}_{0.25} \mathrm{Ba}_{0.15} \mathrm{Sr}_{0.6}\right) \mathrm{TiO}_{3}$ diffusiophoresis nanomotors in deionized water due to concentration difference energy [45]. 
sensing applications, for example, (PAPBA)/Ni/Pt tubular micromotors capture and release yeast cells [41], or molecularly imprinted polymer base catalytic tubular micromotors, which permitted the selective protein transport and isolation [46]. On the other hand, the enhanced fluid convection that is created around the tubular micromotors due to the bubble recoil mechanism has recently been studied and characterized by passive microsphere tracers [49], resulting in a higher mixing efficiency compared to catalytic nanowires or Janus particle motors. Such effect has been closely related to sensing applications, showing the potentiality of using PANI/Pt tubular micromotors for continuous and noninvasive mixing in microarray-based immunosensing applications [50].

The use of template-based micromotors towards environment issues had also been of special importance. Firstly, the viability of using micromotors presenting superhydrophobic surfaces to adsorb and capture oil was demonstrated, opening the door to new remediation approaches for oil-contaminated water [51]. In addition, the oxidative detoxification of organophosphate nerve agents was achieved by using catalytic micromotors in peroxideactivated contaminated samples, which leads to more efficient neutralization processes and shorter reaction times [42]. Although the most extensively used template-based micromotors are the ones which present an inner platinum surface, alternative novel configurations had been recently reported based on polyelectrolyte multilayer tubular nanomotors. Such nanoengines present an easy-tofunctionalize outer surface based on biodegradable natural polysaccharides and platinum nanoparticles assembled by layer-by-layer (LbL) techniques into the template pores of their inner surface (see Figure 4AIV). Their controlled movement was achieved by integrating iron oxide $\left(\mathrm{Fe}_{3} \mathrm{O}_{4}\right)$ nanoparticles, permitting their convenient motion towards HeLa cells and proving their capacity as a drug delivery system by using their outer layers for drug encapsulation [52].

Nanometric tubular micromotors had been recently reported by Zhao et al. [36] opening the doors to scale down the fabrication of such tubular microstructures to the nanoscale by means of electrodeposition techniques. In this particular case, the tubular nanomotors are fabricated using AAO membranes, obtaining tubular bimetallic nanomotors. Firstly, a sacrificial copper layer is deposited, followed by two more deposition steps: one of platinum and one of gold, resulting in a tubular nanometric motor with two defined sections (see Figure 4AIII) [36].

One of the main advantages that the template-based approach offers compared to strain engineering technology is that it is cheap and that it is a mass production method which leads to high-performance catalytic microtubular engines. However, we have to bear in mind that such micromotors will be restricted by the dimensions of the pores of the membrane, becoming specially complicated to tailor their shape and size for specific applications.

\section{Jet propulsion: fuels and motion requirements}

The motion of tubular microjets is due to the presence of certain fuels which promote catalytic reactions on the inner surface of the microjets, inducing the generation and expulsion of bubbles [2,9]. Such micromotors are moving by the bubble recoil mechanism, and their most extended configuration generally presents an inner platinum surface permitting their movement in the presence of hydrogen peroxide and certain surfactants. However, alternative fuels such as water or acids are particularly desirable nowadays, especially concerning their future potential implementation in the biomedical field for drug delivery.

\section{Hydrogen peroxide}

The first reported template-based micromotor moving in the presence of hydrogen peroxide was based on the sequential deposition of PANI, nickel, and platinum, resulting in a highly efficient tubular micromotor [38]. In spite of the toxic nature of the hydrogen peroxide fuel, at low concentrations, many feasible applications had been reported being basically focused on its use as a sensing platform for analytical purposes and its implementation towards environmental issues. Surface tension-reducing agents are crucial components of the fuel for the optimum swimming performance of microjets. Several ionic and non-ionic surfactants accelerate the velocity of microjets because they facilitate the bubble ejection from the microtube [53].

\section{Acidic conditions}

Tubular micromotors are of special interest both for industrial applications and their potential use in medicine, especially in extreme $\mathrm{pH}$ ranges like in the stomach environment. The first micromotor moving in acidic conditions was based on a PANI outer layer and a catalytic zinc inner layer and moved due to the thrust of hydrogen bubbles, showing a sensitive motion-based $\mathrm{pH}$ in extreme conditions (from -0.2 to 1.4) (see Figure 2BI) [43]. An advanced autonomous release system has been recently developed by slightly tuning the design of the PANI/Zn micromotor moving in acidic conditions. In this case, silica and gold nanoparticles were used as model cargo analogues, and they were tightly packed into the double-conical polycarbonate membrane before the zinc electrodeposition took place. After membrane dissolution, fully loaded zinc micromotors that can perform different cargo deliveries at once while they are moving in the presence of extreme acidic conditions were obtained (see Figure 4BII) [44]. 


\section{Water}

Although there is no tubular micromotor reported moving by the bubble recoil mechanism in pure water, alternative mechanisms have offered the possibility of moving such structures in water. On the one hand, diffusion induced the linear and rotational movement of $\left(\mathrm{Pb}_{0.25} \mathrm{Ba}_{0.15} \mathrm{Sr}_{0.6}\right)$ $\mathrm{TiO}_{3}$ nanotube growth in AAO membranes. While one end of the tubes was closed, the opened one was firstly filled with potassium hydroxide solution leading to its motion by means of a diffusion process once immersed in deionized water (see Figure 4C) [45]. On the other hand, tubular motors have been reported moving by the surface-induced Marangoni effect for decontaminating phenolic and azo dye compounds. Such motors were based on commercial pipette tips filled with enzyme solution and surfactant (sodium dodecyl sulfate (SDS)). The spontaneous release of the mixture once it is in the presence of the polluted water results in the movement of the motor, which at the same time releases the bioremediation agent and enhances its interaction with the pollutant due to the effective fluid convection related to its motion [54].

Recent developments have shown that Janus micromotors can be propelled in water, thanks to the water-reactive Al-Ga binary alloy [55] or magnesium [56], serving as inspiration for future alternative propulsion sources of tubular micromotors.

\section{External stimuli}

Recently, it was shown that photoactive, rolled-up titania microtubes can act as micromotors when they are exposed to ultraviolet light [57]. In comparison to the bubblepropelled microjets, the titania microtubes are thought to move by diffusiophoresis [58]. Another external energy source for tubular micromotors is ultrasound. Kagan et al. [59] demonstrated that acoustic droplet vaporization can lead to the displacement of perfluorocarbon-loaded microtubes for targeted tissue penetration and deformation.

\section{Hybrid micromotors: towards biological power sources}

The fact that powerful and durable catalytically driven microjets require toxic fuel for their actuation significantly obstructs their potential in medical and environmental applications and limits them to sensing and diagnostic procedures. There is a vast urge for finding a biocompatible fuel for the microjets which will promote the research field towards the dream of wireless microrobots that can be controlled and moved inside the human body. Using biomolecules and motile cells for the propulsion of microtubes is one first step towards biocompatible solutions for the actuation of devices on the microscale.

\section{Hybrid microjets}

The synergy between the synthetic tubular structures and biological entities gave rise to powerful micromotor configurations. When gold is integrated as an inner nanomembrane, it can likewise be functionalized with enzymes such as catalase that performs the catalytic reaction with hydrogen peroxide more efficiently than Pt. It requires lower concentrations of fuel for the locomotion of the microtubes. The first reported micromotor moving by means of $\mathrm{H}_{2} \mathrm{O}_{2}$ decomposition by catalase was based on rolled-up microtubes whose inner gold surface was functionalized by self-assembling monolayers, permitting the covalent immobilization of catalase. The micromotor moved at fairly low concentrations of $\mathrm{H}_{2} \mathrm{O}_{2}(1.5 \% \mathrm{w} / \mathrm{w})$ by bubble recoil, showing a trajectory based on turns and circles due to variable viscous drag forces (Figure 5AI) [15]. The first application of catalase-based micromotors was reported by a similar configuration based on template-based micromotors (PEDOT)/Au-catalase, being used in toxicity assays for water assessment. The efficiency of the catalase enzyme was attenuated in the presence of certain toxins, inhibiting its activity and leading to lower speed and lifetime. Such behavior could be correlated to the concentration of certain pollutants of interest, leading to environmentally friendly assays which avoid the use of living aquatic organisms for water quality testing (Figure 5AII) [60].

\section{Spermbots}

Biological motors or motile cells are attractive sources for actuation of microstructures $[61,64,65]$ and generally target the development of new tools for minimal invasive surgery, diagnostics, and drug delivery. Biological motors are attractive as driving sources because of their biocompatibility, autonomous motion, and high efficiency. Recently, a new generation of tubular micromotors was invented by harnessing motile spermatozoa as propulsion force for rolled-up microtubes (spermbots) [63]. This biocompatible approach circumvents the usage of toxic fuels and offers the motion of micromotors under physiological conditions. In the case of the spermbot, motile bovine spermatozoa are trapped inside 50- $\mu$ m-long microtubes that have a diameter that is slightly larger than the head of the sperm cell. The flagellum serves as driving force that propels the microtube forward. Since the microtube consists of iron nanomembranes, it can be steered externally by magnetic fields (Figure 5BI) [63]. It was recently demonstrated that a setup of electromagnetic coils can be used in combination with an optical microscope to perform closed-loop control of the spermbots. Image recognition allows the selection of a sperm-driven tube and the targeted delivery to a selected reference point [62], being the first example of wirelessly controlling a non-magnetic cell by a magnetic field (Figure 5BII).

The spermbot has potential for biomedical applications and opens up a new field of use for micromotors: the spermbot can serve as an explorative tool in reproductive technologies to study infertility and develop 


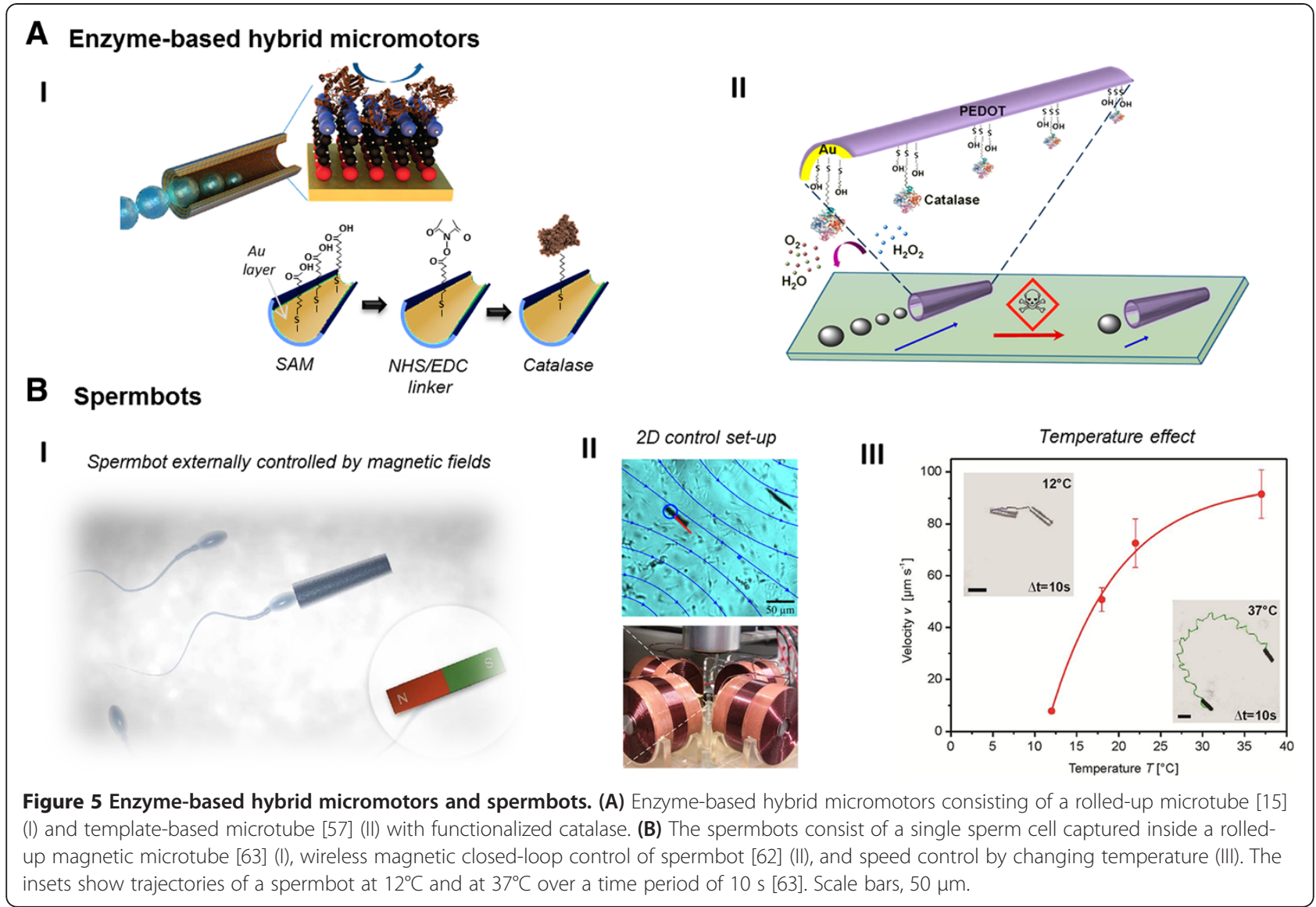

new assisted in vivo reproduction techniques. However, certain requirements need to be accomplished for the application in this field: the sperm cell needs to maintain its motility and fertilization capability throughout the whole delivery process. In addition, a proper cell release mechanism needs to be applied to deliver the sperm cell to the fertilization site as well as suitable imaging and control techniques. There are several challenges that must be addressed in future studies when harnessing spermatozoa as transporters, some of which were discussed in a recent editorial [66]. One of the challenges that spermbots will be facing when used as drug delivery vehicles is the immune response of the host organism. The sperm cell as a foreign cell to the body might be engulfed by leukocytes. This process of phagocytosis will limit the lifetime of the spermbots. However, the microtube as an envelope for the sperm cell can be equipped in ways to prevent the leukocytosis in the same fashion that bacterial pathogens are able to overcome the phagocytotic engulfment and killing by appropriate blocking methods [67].

\section{Conclusions}

There have been significant advances during the last decade in the development of tubular micromotors. Both fabrication methods are well established and enable the fast and reliable mass production of microtubes. Methods to implement further functionalities in the tubes, such as the integration of enzymes or polymers, have been demonstrated. Hydrogen peroxide as fuel is currently the limiting factor for applications and further development of the catalytically driven jets. The use of alternative biocompatible fuels that are inherently present in the physiological environment is of special interest to promote their motion. Many proof-of-concept applications on the microscale demonstrate the potential of micromotors in the field of environmental science, sensing, and medical intervention. Hybrid micromotors, such as spermbots, offer an alternative approach to tubular micromotors and at the same time open up a new field of potential impact in reproduction research and in the fight against infertility by targeted delivery of sperm cells to the fertilization site.

\section{Abbreviations}

3D: three-dimensional; $\mathrm{AAO}$ : aluminum oxide; $\mathrm{Fe}_{3} \mathrm{O}_{4}$ : iron oxide; $\mathrm{H}_{2} \mathrm{O}_{2}$ : hydrogen peroxide; LbL: layer-by-layer; PANI: polyaniline; PEDOT: poly(3,4-ethylenedioxythiophene); PPy: polypyrrole; PAPBA: poly(3aminophenylboronic acid); SAMs: self-assembling monolayers; SDS: sodium dodecyl sulfate.

\section{Competing interests}

The authors declare that they have no competing interests. 


\section{Authors' contributions}

VM, MG, and OGS conceived and designed the manuscript. VM and MG wrote the draft. All authors read and approved the final manuscript.

\section{Acknowledgements}

The authors thank the Volkswagen Foundation (\# 86 362) and the DFG Priority Programme (SPP 1726). VM thanks B. Koch for the fruitful discussion.

\section{Author details}

'Institute for Integrative Nanosciences, Leibniz Institute for Solid State and Materials Research Dresden, 01069 Dresden, Germany. ${ }^{2}$ Material Systems for Nanoelectronics, Chemnitz University of Technology, 09126 Chemnitz, Germany. ${ }^{3}$ Center for Advancing Electronics Dresden, Dresden University of Technology, 01187 Dresden, Germany.

\section{Received: 30 June 2014 Accepted: 22 September 2014}

\section{Published online: 08 October 2014}

\section{References}

1. Schmidt OG, Eberl K (2001) Nanotechnology: thin solid films roll up into nanotubes. Nature 410:168

2. Mei YF, Huang GS, Solovev AA, Bermúdez Ureña E, Moench I, Ding F, Reindl T, Fu RKY, Chu PK, Schmidt OG (2008) Versatile approach for integrative and functionalized tubes by strain engineering of nanomembranes on polymers. Adv Mater 20:4085-4090

3. Martinez-Cisneros CS, Sanchez S, Xi W, Schmidt OG (2014) Ultracompact three-dimensional tubular conductivity microsensors for ionic and biosensing applications. Nano Lett 14:2219-2224

4. Vervacke C, Bof Bufon CC, Thurmer DJ, Schmidt OG (2014) Threedimensional chemical sensors based on rolled-up hybrid nanomembranes. RSC Adv 4:9723-9729

5. Sun X, Yan C, Chen Y, Si W, Deng J, Oswald S, Liu L, Schmidt OG (2014) Three-dimensionally "curved" NiO nanomembranes as ultrahigh rate capability anodes for Li-ion batteries with long cycle lifetimes. Adv Energy Mater doi:10.1002/aenm.201300912

6. Grimm D, Bof Bufon CC, Deneke C, Atkinson P, Thurmer DJ, Schäffel F, Gorantla S, Bachmatiuk A, Schmidt OG (2013) Rolled-up nanomembranes as compact 3D architectures for field effect transistors and fluidic sensing applications. Nano Lett 13:213-218

7. Koch B, Sanchez S, Schmidt CK, Swiersy A, Jackson SP, Schmidt OG (2014) Confinement and deformation of single cells and their nuclei inside sizeadapted microtubes. Adv Healthcare Mater doi:10.1002/adhm.201300678

8. Xi W, Schmidt CK, Sanchez S, Gracias DH, Carazo-Salas RE, Jackson SP, Schmidt OG (2014) Rolled-up functionalized nanomembranes as three-dimensional cavities for single cell studies. Nano Lett 14:4197-4204

9. Solovev AA, Mei Y, Urena EB, Huang G, Schmidt OG (2009) Catalytic microtubular jet engines self-propelled by accumulated gas bubbles. Small 5:1688-1692

10. Solovev AA, Xi W, Gracias DH, Harazim SM, Deneke SM, Sanchez S, Schmidt OG (2012) Self-propelled nanotools. ACS Nano 6:1751-1756

11. Mönch I, Makarov D, Koseva R, Baraban L, Karnaushenko D, Kaiser C, Arndt KF, Schmidt OG (2011) Rolled-up magnetic sensor: nanomembrane architecture for in-flow detection of magnetic objects. ACS Nano 5:7436-7442

12. Li J, Zhang J, Gao W, Huang G, Di Z, Liu R, Wang J, Mei Y (2013) Dry-released nanotubes and nanoengines by particle-assisted rolling. Adv Mater 25:3715-3721

13. Harazim SM, Bolanos Quinones VA, Kiravittaya S, Sanchez S, Schmidt OG (2012) Lab-in-a-tube: on-chip integration of glass optofluidic ring resonators for label-free sensing applications. Lab Chip 12:2649-2655

14. Harazim SM (2012) Rolled-up microtubes as components for lab-on-a-chip devices. Technische Universität Chemnitz, Dissertation

15. Sanchez S, Solovev AA, Mei Y, Schmidt OG (2010) Dynamics of biocatalytic microengines mediated by variable friction control. J Am Chem Soc 132:13144-13145

16. Sanchez S, Solovev AA, Schulze S, Schmidt OG (2011) Controlled manipulation of multiple cells using catalytic microbots. Chem Commun 47:698-700

17. Kagan D, Campuzano S, Balasubramanian S, Kuralay F, Flechsig GU, Wang J (2011) Functionalized micromachines for selective and rapid isolation of nucleic acid targets from complex samples. Nano Lett 11:2083-2087
18. Balasubramanian S, Kagan D, Hu CMJ, Campuzano S, Lobo-Castañon MJ, Lim N, Kang DY, Zimmerman M, Zhang L, Wang J (2011) Micromachine-enabled capture and isolation of cancer cells in complex media. Angew Chem Int Ed 50:4161-4164

19. Orozco J, Campuzano S, Kagan D, Zhou M, Gao W, Wang J (2011) Dynamic isolation and unloading of target proteins by aptamer-modified microtransporters. Anal Chem 83:7962-7969

20. Zhao G, Sanchez S, Schmidt OG, Pumera M (2012) Micromotors with built-in compasses. Chem Commun 48:10090-10092

21. Xi W, Solovev AA, Ananth AN, Gracias DH, Sanchez S, Schmidt OG (2013) Rolled-up magnetic microdrillers: towards remotely controlled minimally invasive surgery. Nanoscale 5:1294-1297

22. Sanchez S, Solovev AA, Harazim SM, Schmidt OG (2011) Microbots swimming in the flowing streams of microfluidic channels. J Am Chem Soc 133:701-703

23. Khalil ISM, Magdanz V, Sanchez S, Schmidt OG, Misra S (2014) The control of self-propelled microjets inside a microchannel with time-varying flow rates. IEEE Trans Robot 30:49-58

24. Khalil ISM, Magdanz V, Sanchez S, Schmidt OG, Misra S (2014) Wireless magneticbased closed-loop control of self-propelled microjets. PLoS One 9:e83053

25. Khalil ISM, Magdanz V, Sanchez S, Schmidt OG, Misra S (2013) Threedimensional closed-loop control of self-propelled microjets. Appl Phys Lett 103:172404-4

26. Solovev AA, Smith EJ, Bof Bufon CC, Sanchez S, Schmidt OG (2011) Lightcontrolled propulsion of catalytic microengines. Angew Chem Int Edit 50:10875-10878

27. Sanchez S, Ananth AN, Fomin VM, Viehrig M, Schmidt OG (2011) Superfast motion of catalytic microjet engines at physiological temperature. J Am Chem Soc 133:14860-14863

28. Soler LC, Martínez-Cisneros CS, Swiersy A, Sánchez S, Schmidt OG (2013) Thermal activation of catalytic microjets in blood samples using microfluidic chips. Lab Chip 13:4299-4303

29. Soler L, Magdanz V, Fomin VM, Sanchez S, Schmidt OG (2013) Self-propelled micromotors for cleaning polluted water. ACS Nano 7:9611-9620

30. Restrepo-Pérez L, Soler L, Martínez-Cisneros CS, Sánchez S, Schmidt OG (2014) Trapping self-propelled micromotors with microfabricated chevron and heart-shaped chips. Lab Chip 14:1515-1518

31. Restrepo-Pérez L, Soler L, Martínez-Cisneros CS, Sánchez S, Schmidt OG (2014) Biofunctionalized self-propelled micromotors as an alternative on-chip concentrating system. Lab Chip 14:2914-2917

32. Mei YF, Solovev AA, Sanchez S, Schmidt OG (2011) Rolled-up nanotech on polymers: from basic perception to self-propelled catalytic microengines. Chem Soc Rev 40:2109-2119

33. Stoychev G, Zakharchenko S, Turcaud S, Dunlop JWC, Ionov L (2012) Shape-programmed folding of stimuli-responsive polymer bilayers. ACS Nano 6:3925-3934

34. Magdanz V, Stoychev G, Magdanz V, Stoychev G, lonov L, Sanchez S, Schmidt OG (2014) Stimuli-responsive microjets with reconfigurable shape. Angew Chem Int Edit 126:2711-2715

35. Manesh KM, Cardona M, Yuan R, Clark M, Kagan D, Balasubramanian S, Wang J (2010) Template-assisted fabrication of salt-independent catalytic tubular microengines. ACS Nano 4:1799-1804

36. Zhao G, Ambrosi A, Pumera M (2013) Self-propelled nanojets via template electrodeposition. Nanoscale 5:1319-1324

37. Gao W, Sattayasamitsathit S, Uygun A, Pei A, Ponedal A, Wang J (2012) Polymer-based tubular microbots: role of composition and preparation. Nanoscale 4:2447-2453

38. Gao W, Sattayasamitsathit S, Orozco J, Wang J (2011) Highly efficient catalytic microengines: template electro-synthesis of polyaniline-platinum microtubes. J Am Chem Soc 133:11862-11864

39. Zhao G, Pumera M (2013) Concentric bimetallic microjets by electrodeposition. RSC Adv 3:3963-3966

40. Garcia M, Orozco J, Guix M, Gao W, Sattayasamitsathit S, Escarpa A, Merkoc A, Wang J (2013) Micromotor-based lab-on-chip immunoassays. Nanoscale 5:1325-1331

41. Kuralay F, Sattayasamitsathit S, Gao W, Uygun A, Katzenberg A, Wang J (2012) Self-propelled carbohydrate-sensitive microtransporters with 'built-in' boronic-acid recognition for isolating sugars and cells. J Am Chem Soc 134:15217-15220

42. Orozco J, Cheng G, Vilela D, Sattayasamitsathit S, Vazquez-Duhalt R, Valdés-Ramírez G, Pak OS, Escarpa A, Kan C, Wang J (2013) Micromotor- 
based high-yielding fast oxidative detoxification of chemical threats. Angew Chem Int Ed 52:13276-13279

43. Gao W, Uygun A, Wang J (2012) Hydrogen-bubble propelled zinc-based microrockets in strongly acidic media. J Am Chem Soc 134:897-900

44. Sattayasamitsathit S, Kou H, Gao W, Thavarajah W, Kaufmann K, Zhang L, Wang J (2014) Fully-loaded micromotors for combinatorial delivery and autonomous release of cargoes. Small 10:2830-2833

45. Liu W, He R, Zhu H, Hu H, Li M, Zhao XZ (2010) Ultrafast nanotube based diffusiophoresis nanomotors. Appl Phys Lett 96:053114-053117

46. Orozco J, Cortés A, Cheng G, Sattayasamitsathit S, Gao W, Feng X, Shen Y, Wang J (2013) Molecularly imprinted polymer-based catalytic micromotors for selective protein transport. J Am Chem Soc 135:5336-5339

47. Xu T, Soto F, Gao W, Garcia-Gradilla V, Li J, Zhang X, Wang J (2014) Ultrasound-modulated bubble propulsion of chemically powered microengines. J Am Chem Soc 136:8552-8555

48. Campuzano S, Orozco J, Kagan D, Guix M, Gao W, Sattayasamitsathit S, Claussen JC, Merkoçi A, Wang J (2012) Bacterial isolation by lectin-modified microengines. Nano Lett 12:396-401

49. Orozco J, Jurado-Sánchez B, Wagner G, Gao W, Vazquez-Duhalt R, Sattayasamitsathit S, Galarnyk M, Cortés A, Saintillan D, Wang J (2014) Bubble-propelled micromotors for enhanced transport of passive tracers. Langmuir 30:5082-5087

50. Morales-Narváez E, Guix M, Medina-Sánchez M, Mayorga-Martinez CC, Merkoçi A (2014) Micromotor enhanced microarray technology for protein detection. Small 10:2542-2548

51. Guix M, Orozco J, Garcia M, Gao W, Sattayasamitsathit S, Merkoci A, Escarpa A, Wang J (2012) Superhydrophobic alkanethiol-coated microsubmarines for effective removal of oil. ACS Nano 6:4445-4451

52. Wu Z, Wu Y, He W, Lin X, Sun J, He Q (2013) Self-propelled polymer-based multilayer nanorockets for transportation and drug release. Angew Chem Int Ed 52:7000-7003

53. Simmchen J, Magdanz V, Sanchez S, Chokmaviroj S, Ruiz-Molina D, Baeza A, Schmidt OG (2014) Effect of surfactants on the performance of tubular and spherical micromotors - a comparative study. RSC Adv 4:20334-20340

54. Orozco J, Vilela D, Valdés-Ramírez G, Fedorak Y, Escarpa A, Vazquez-Duhalt R, Wang J (2014) Efficient biocatalytic degradation of pollutants by enzymereleasing self-propelled motors. Chem Eur J 20:2866-2871

55. Gao W, Pei A, Wang J (2012) Water-driven micromotors. ACS Nano 6:8432-8438

56. Gao W, Feng X, Pei A, Gu Y, Li J, Wang J (2013) Seawater-driven magnesium based Janus micromotors for environmental remediation. Nanoscale 5:4696-4700

57. Giudicatti S, Marz SM, Soler L, Madani A, Jorgensen MR, Sanchez S, Schmidt OG (2014) Photoactive rolled-up $\mathrm{TiO}_{2}$ microtubes: fabrication, characterization and applications. J Mater Chem C 2:5892-5901

58. Hong YY, Diaz M, Cordova-Figueroa UM, Sen A (2010) Light-driven titaniumdioxide-based reversible microfireworks and micromotor/micropump systems. Adv Funct Mater 20:1568-1576

59. Kagan D, Benchimol MJ, Claussen JC, Chuluun-Erdene E, Esener S, Wang J (2012) Acoustic droplet vaporization and propulsion of perfluorocarbonloaded microbullets for targeted tissue penetration and deformation. Angew Chem Int Ed 51:7519-7522

60. Orozco J, García-Gradilla V, D'Agostino M, Gao W, Cortés A, Wang J (2013) Artificial enzyme-powered microfish for water-quality testing. ACS Nano 7:818-824

61. Martel S (2012) Bacterial microsystems and microrobots. Biomed Microdevices 14:1033-1045

62. Khalil ISM, Magdanz V, Sanchez S, Schmidt OG, Misra S (2014) Biocompatible, accurate, and fully autonomous: a sperm-driven micro-bio-robot. J Micro-Bio Robot 9:79-86

63. Magdanz V, Sanchez S, Schmidt OG (2013) Development of a sperm-flagella driven micro-bio-robot. Adv Mater 25:6581-6588

64. Behkam B, Sitti M (2007) Bacterial flagella-based propulsion and on/off motion control of microscale objects. Appl Phys Lett 90:23902-23904

65. Soong RK, Bachand GD, Neves HP, Olkhovets AG, Craighead HG, Montemagno CD (2000) Powering an inorganic nanodevice with a biomolecular motor. Science 290:1555-1558
66. Magdanz V, Schmidt OG (2014) Spermbots: potential impact for drug delivery and assisted reproductive technologies. Expert Opin Drug Deliv 11:1125-1129

67. Hornef MW, Wick MJ, Rhen M, Normack S (2002) Bacterial strategies for overcoming host innate and adaptive immune responses. Nat Immunol 3:1033-1040

doi:10.1186/s40638-014-0011-6

Cite this article as: Magdanz et al: Tubular micromotors: from microjets to spermbots. Robotics and Biomimetics 2014 1:11

\section{Submit your manuscript to a SpringerOpen ${ }^{\odot}$ journal and benefit from:}

- Convenient online submission

- Rigorous peer review

- Immediate publication on acceptance

- Open access: articles freely available online

High visibility within the field

- Retaining the copyright to your article

Submit your next manuscript at $\gg$ springeropen.com 